\title{
Fredholm determinants and the statistics of charge transport
}

\section{Journal Article}

Author(s):

Avron, J. E.; Bachmann, S.; Graf, G. M.; Klich, I.

Publication date:

2007

Permanent link:

https://doi.org/10.3929/ethz-b-000007323

Rights / license:

In Copyright - Non-Commercial Use Permitted

Originally published in:

Communications in Mathematical Physics 280(3), https://doi.org/10.1007/s00220-008-0449-x 


\title{
Fredholm Determinants and the Statistics of Charge Transport
}

\author{
J. E. Avron ${ }^{1}$, S. Bachmann ${ }^{2}$, G. M. Graf ${ }^{2}$, I. Klich ${ }^{3}$ \\ 1 Department of Physics, Technion, 32000 Haifa, Israel \\ 2 Theoretische Physik, ETH-Hönggerberg, 8093 Zürich, Switzerland. E-mail: gmgraf@itp.phys.ethz.ch \\ 3 Condensed Matter Department, Caltech, MC 114-36, Pasadena, CA 91125, USA
}

Received: 1 May 2007 / Accepted: 20 August 2007

Published online: 13 March 2008 - C Springer-Verlag 2008

\begin{abstract}
Using operator algebraic methods we show that the moment generating function of charge transport in a system with infinitely many non-interacting Fermions is given by a determinant of a certain operator in the one-particle Hilbert space. The formula is equivalent to a formula of Levitov and Lesovik in the finite dimensional case and may be viewed as its regularized form in general. Our result embodies two tenets often realized in mesoscopic physics, namely, that the transport properties are essentially independent of the length of the leads and of the depth of the Fermi sea.
\end{abstract}

\section{Introduction}

Models of physical systems are often formulated with the help of one or a few parameters which guarantee that whatever one computes is well defined and finite while, at the same time, are believed not to affect properties of physical interest. Examples are: the number of particles in a macroscopic system, and the lattice spacing (ultraviolet cutoffs) in the study of critical phenomena.

The theory of transport in mesoscopic systems has two such parameters: the length of the incoming leads that connect to the system and the depth of the Fermi sea. The independence of the length of the leads is the statement that well designed experiments measure the transport properties of the mesoscopic system and are independent of the measuring circuit. The independence of the depth of the Fermi sea expresses the irrelevance for transport of electrons that are buried deep in the Fermi sea, since in most situations they can not be excited above it. In this sense there is freedom from both the volume and the ultraviolet scale. See [21] for a numerical investigation of these properties.

One strategy to address this type of behavior is to consider idealized systems where the parameters are taken to be infinitely large. The limiting idealized system comes with the price tag that expressions for physical quantities that are otherwise guaranteed to be finite, may become ambiguous, formal and even infinite. The value in worrying about 
this idealized, possibly un-physical system, is precisely in that once the ambiguities and infinities are resolved, they teach us something important about the finite physical model, namely, that the parameters used in its formulation, do indeed effectively disappear from the physical properties. Their role is effectively reduced to the control of the small differences between the idealized model and the physical one.

We shall consider a problem of this kind that arises in the context of modeling the statistics of charge transport from one reservoir to another. Levitov and Lesovik [13] wrote a formula for the appropriate generating function in terms of a certain infinite dimensional determinant. The formula has found a number of applications to shot and thermal noise in devices like transmission barriers, cavities, and interfaces. When one wants to apply this formula to the idealized cases one finds ambiguities and, as emphasized by Levitov et al. [8,11,12], the determinant requires proper definition through regularization. We intend to further the understanding of these points by providing an alternative, mathematically consistent, form for the determinant. As we shall see, the "regularized form" of the determinant naturally emerges once the quantum dynamics is formulated on the state space of the idealized system.

In the next section we introduce the statistics of charge transport, review the LevitovLesovik determinant, and propose a regularization. In Sect. 3 we state the main results. Section 4 is devoted to proofs and begins with a short overview thereof. Finally, Sect. 5 exemplifies the assumptions made in this work.

\section{The Levitov-Lesovik Formula and Its Regularization}

We consider a lead, where independent electrons are evolved over some time interval and ask about the statistics of the charge transferred from the left to the right portion of the lead. To begin, we recall the result obtained in [13] and further elaborated in $[12,8]$. We present its derivation and generalization to finite times along the heuristic lines given in [10], in the sense that we do as if the one-particle Hilbert space $\mathcal{H}$ were finite-dimensional.

The fermionic Fock space $\mathcal{F}$ over $\mathcal{H}$ contains a distinguished state, the vacuum, with the physical interpretation of a no particle state. Let Tr, resp. tr, denote the trace on $\mathcal{F}$, resp. $\mathcal{H}$. Let $U$ be the unitary on $\mathcal{H}$ representing the time evolution, and $Q$ the projection corresponding to the right portion of the lead. Their second quantizations,

$\Gamma(U)=\wedge_{i=1}^{k} U_{i}$, resp. $\mathrm{d} \Gamma(Q)=\sum_{i=1}^{k} Q_{i}$ on $k$-particle states, then stand for the evolution on $\mathcal{F}$, resp. for the charge in that portion. We suppose that the initial many particle (mixed) state is of the form

$$
P=Z^{-1} \Gamma(M)
$$

for some operator $M \geq 0$, where $Z=\operatorname{Tr} \Gamma(M)=\operatorname{det}(1+M)$ ensures that $\operatorname{Tr} P=1$. The reduced one-particle density matrix $N$ is defined by the property that

$$
\operatorname{tr}(A N)=\operatorname{Tr}(\mathrm{d} \Gamma(A) P)
$$

for any one-particle operator $A$ on $\mathcal{H}$. In our case, $N=M(1+M)^{-1}$. This follows from

$$
\begin{aligned}
\operatorname{Tr}\left(\mathrm{e}^{\mathrm{i} \lambda \mathrm{d} \Gamma(A)} P\right) & =\operatorname{Tr}\left(\Gamma\left(\mathrm{e}^{\mathrm{i} \lambda A}\right) P\right)=Z^{-1} \operatorname{Tr}\left(\Gamma\left(\mathrm{e}^{\mathrm{i} \lambda A} M\right)\right)=\frac{\operatorname{det}\left(1+\mathrm{e}^{\mathrm{i} \lambda A} M\right)}{\operatorname{det}(1+M)} \\
& =\operatorname{det}\left(1-N+\mathrm{e}^{\mathrm{i} \lambda A} N\right)
\end{aligned}
$$

by taking the derivative at $\lambda=0$. 
In the following, we assume that $M$ and $Q$, and hence $P$ and $\mathrm{d} \Gamma(Q)$, commute, which physically means that in the state defined by $P$, charge in the lead measured by $\mathrm{d} \Gamma(Q)$ is a good quantum number. Hence

$$
P|\alpha\rangle=\rho_{\alpha}|\alpha\rangle, \quad \mathrm{d} \Gamma(Q)|\alpha\rangle=n_{\alpha}|\alpha\rangle,
$$

for some basis $\{|\alpha\rangle\}$ of $\mathcal{F}$. The moment generating function for the charge transfer statistics is

$$
\chi(\lambda)=\sum_{n \in \mathbb{Z}} p_{n} \mathrm{e}^{\mathrm{i} \lambda n}
$$

where $p_{n}$ is the probability for $n$ electrons being deposited into the right portion of the lead by the end of the time interval. It may be computed as a sum over initial and final states, $\alpha$ resp. $\beta$, with the former weighted according to their probabilities $\rho_{\alpha}$ :

$$
\begin{aligned}
\chi(\lambda) & =\sum_{\alpha, \beta}|\langle\beta|\Gamma(U)| \alpha\rangle|^{2} \rho_{\alpha} \mathrm{e}^{\mathrm{i} \lambda\left(n_{\beta}-n_{\alpha}\right)}=\operatorname{Tr}\left(\Gamma(U)^{*} \mathrm{e}^{\mathrm{i} \lambda \mathrm{d} \Gamma(Q)} \Gamma(U) \mathrm{e}^{-\mathrm{i} \lambda \mathrm{d} \Gamma(Q)} P\right) \\
& =Z^{-1} \operatorname{Tr}\left(\Gamma\left(U^{*} \mathrm{e}^{\mathrm{i} \lambda Q} U \mathrm{e}^{-\mathrm{i} \lambda Q} M\right)\right)=\operatorname{det}\left(1-N+\mathrm{e}^{\mathrm{i} \lambda U^{*} Q U} N \mathrm{e}^{-\mathrm{i} \lambda Q}\right),
\end{aligned}
$$

where the trace has been computed in the basis $|\alpha\rangle$, with an identity $\sum|\beta\rangle\langle\beta|=1$ absorbed at the left of $\Gamma(U)$; the last equality is by (1). This is the Levitov-Lesovik formula:

$$
\chi(\lambda)=\operatorname{det} D(\lambda), \quad D(\lambda)=N^{\prime}+\mathrm{e}^{\mathrm{i} \lambda Q_{U}} N \mathrm{e}^{-\mathrm{i} \lambda Q},
$$

with $N^{\prime}=1-N$ and $Q_{U}=U^{*} Q U$. Since $Q$ is a projection, $\mathrm{e}^{2 \pi \mathrm{i} Q}=\mathrm{e}^{2 \pi \mathrm{i} Q_{U}}=1$ and $D(\lambda)$ is a periodic function with period $2 \pi$. This expresses the integrality of charge transport.

An example of a state of interest is that of a system at inverse temperature $\beta$ having one-particle Hamiltonian $H$; it is $P=Z^{-1} \Gamma(M)$ with $M=\exp (-\beta H)$ and $N=$ $[1+\exp (\beta H)]^{-1}$. In the limit $\beta \rightarrow \infty, P$ describes the Fermi sea, whence $N$ is the projection onto the occupied one-particle states.

The above derivation would be rigorous if the one-particle Hilbert space were finite dimensional. The question we want to address here is what is the correct replacement for $D(\lambda)$ when $P$ describes infinitely many particles, both because the lead may be infinitely extended spatially (as appropriate for an open system) and because the Fermi sea may be very or even infinitely deep. The first concern appears to affect only the derivation, but not the result, Eq. (3). However, by the second, $D(\lambda)$ differs from the identity by more than a trace class operator, as would be required by the definition of a Fredholm determinant. A manifestation thereof (and in a sense the only one) is that the expected charge transport

$$
\langle n\rangle=-\mathrm{i} \chi^{\prime}(0)=-\left.\mathrm{i} \frac{d}{d \lambda} \operatorname{det} D(\lambda)\right|_{\lambda=0}=\operatorname{tr}\left(\left(Q_{U}-Q\right) N\right)
$$

involves an operator which is not trace class in the stated situation. These statements are illustrated (in the $\beta=\infty$ case) in Fig. 1 representing the phase space of a single particle moving freely.

The Fermi sea $N$ corresponds to $|p|<p_{F}, p_{F}$ being the Fermi momentum, and similarly the right half of the lead $Q$ to $x>0$. The free evolution, which we take 

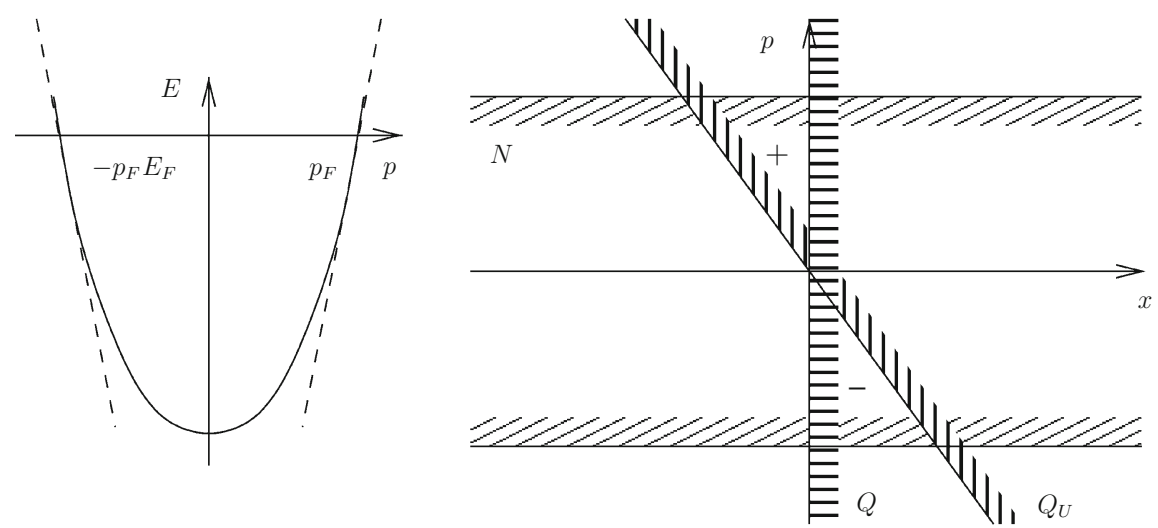

Fig. 1. Left: Dispersion relation $E(p)$ of free particles, and its linearization. Right: Phase space (coordinates $x, p$ ) with regions selected by $N, Q$ and $Q_{U}$, and hatched along their boundaries with slanted, horizontal, and vertical dashes, respectively

as a simple example for $U$, is a horizontal shear, so that $Q_{U}-Q$ is associated with two sectors, labelled + and - . Their intersection with the horizontal strip associated with $N$ delineates the phase space support of $\left(Q_{U}-Q\right) N$. Its area, which is a rough estimate of the trace class norm of the operator, is proportional to the depth of the sea. If the dispersion relation is conveniently linearized at $\pm p_{F}$, the depth becomes infinite, implying that the operator is not trace class. As a remedy, we note that the expression

$$
\operatorname{tr}\left(Q N-Q_{U} N_{U}\right)=0,
$$

with $N_{U}=U^{*} N U$, vanishes by splitting the trace, though only suggestively so, because the traces fail to exist separately due to the infinite spatial extent of the leads. Adding nevertheless that expression to (4) yields

$$
\langle n\rangle=\operatorname{tr}\left(Q_{U}\left(N-N_{U}\right)\right),
$$

which vanishes in the special case of the free evolution, $N_{U}=N$, and is expected to be finite in others. This way of renormalizing the expression is actually declaring that the Fermi sea does not contribute to the current, instead of relying on a compensation between left and right movers, as indicated by + and - in the figure.

This heuristic manipulation motivates the following regularization of the LevitovLesovik determinant. Replacing $D(\lambda)$ by

$$
\widetilde{D}(\lambda)=\mathrm{e}^{-\mathrm{i} \lambda N_{U} Q_{U}} D(\lambda) \mathrm{e}^{\mathrm{i} \lambda N Q}
$$

should not change the value of the determinant, since informally

$$
\operatorname{det}\left(\mathrm{e}^{-\mathrm{i} \lambda N_{U} Q_{U}}\right) \cdot \operatorname{det}\left(\mathrm{e}^{\mathrm{i} \lambda N Q}\right)=\mathrm{e}^{\mathrm{i} \lambda \operatorname{tr}\left(Q N-Q_{U} N_{U}\right)}=1 .
$$

Incidentally, this regularization affects only the first cumulant of the statistics, i.e. the average charge transfer, since the full set of cumulants is generated by $\log \operatorname{det} \widetilde{D}(\lambda)$. We are thus led to recast Eq. (3) as

$$
\begin{aligned}
& \chi(\lambda)=\operatorname{det} \widetilde{D}(\lambda), \\
& \widetilde{D}(\lambda)=\mathrm{e}^{-\mathrm{i} \lambda N_{U} Q_{U}} N^{\prime} \mathrm{e}^{\mathrm{i} \lambda N Q}+\mathrm{e}^{\mathrm{i} \lambda N_{U}^{\prime} Q_{U}} N \mathrm{e}^{-\mathrm{i} \lambda N^{\prime} Q} .
\end{aligned}
$$


It is to be noted that this representation of $\chi(\lambda)$ is manifestly particle-hole symmetric:

$$
\chi_{N}(\lambda)=\chi_{N^{\prime}}(-\lambda) .
$$

It is also $2 \pi$-periodic in $\lambda$, though manifestly so only at $T=0$ since $N Q, N^{\prime} Q$ etc. are all projections. In that case, Eq. (9) reduces to

$$
\widetilde{D}(\lambda)=1+Q_{U}\left(N-N_{U}\right)\left(\left(\mathrm{e}^{\mathrm{i} \lambda}-1\right) N-\left(\mathrm{e}^{-\mathrm{i} \lambda}-1\right) N^{\prime}\right),
$$

which shows that the generating function $\chi(\lambda)$ is well-defined whenever its first cumulant (5) is. As we shall see, a slightly weaker result holds at positive temperature.

Let us mention a few connections to other works. A related regularization of the Levitov-Lesovik determinant at zero temperature was used in [15], where the relation of counting statistics to a Riemann-Hilbert problem was studied. Another one, exhibiting the symmetry (10), was proposed in [16]. On the more mathematical side, regularizations of determinants have been related to renormalization in [22], though by means of a somewhat different regularization known as $\operatorname{det}_{n}(1+A)=\operatorname{det}(1+$ $A) \exp \left(\operatorname{tr} \sum_{j=1}^{n-1}(-1)^{j} A^{j} / j\right)$. The role of $\mathrm{C}^{*}$-algebras in the theory of open systems has recently been advocated by Jakšić and Pillet, see e.g. [9], in general, but also to fluctuations in particular. A generating function for fluctuations of energy in bosonic systems has been proposed by [19].

The purpose of this work is to show that, under reasonable assumptions, Eq. (8) is obtained without recourse to regularizations, if the second quantization is built upon the Fermi sea rather than on the vacuum $N=0$.

\section{Results}

Let $\mathcal{H}$ be a separable Hilbert space with the following operators acting on it: An orthogonal projection $Q$, a unitary $U$, and a selfadjoint $N$, with

$$
0 \leq N \leq 1
$$

whose physical interpretations have been described in the previous section. Let $N^{\prime}=$ $1-N$. We denote by $\mathcal{I}_{p},(p \geq 1)$ the Schatten trace ideals, i.e. the space of all bounded operators $A$ on $\mathcal{H}$ such that $\|A\|_{p}^{p}:=\operatorname{tr}|A|^{p}<\infty$.

The algebra of canonical anticommutation relations (CAR) over $\mathcal{H}$ is the $\mathrm{C}^{*}$-algebra $\mathcal{A}(\mathcal{H})$ generated by 1 , and the elements $a(f)$ and $a^{*}(f),(f \in \mathcal{H})$, such that

i. the map $f \longmapsto a(f)$ is antilinear,

ii. $a^{*}(f)=a(f)^{*}$,

iii. these elements satisfy the following anticommutation relations:

$$
\left\{a(f), a^{*}(g)\right\}=(f, g) 1,
$$

all other anticommutators vanishing.

A (global) gauge transformation is expressed by the automorphism $\alpha_{\lambda}: a(f) \mapsto$ $a\left(\mathrm{e}^{\mathrm{i} \lambda} f\right)$. A state $\omega$ on $\mathcal{A}(\mathcal{H})$ is gauge-invariant if $\omega\left(\alpha_{\lambda}(A)\right)=\omega(A)$ for all $A \in \mathcal{A}(\mathcal{H})$. The operator $N$ defines a gauge-invariant quasi-free state $\omega_{N}$ through

$$
\omega_{N}\left(a^{*}\left(f_{n}\right) \ldots a^{*}\left(f_{1}\right) a\left(g_{1}\right) \ldots a\left(g_{m}\right)\right)=\delta_{n m} \operatorname{det}\left(g_{i}, N f_{j}\right),
$$


or equivalently by $\omega_{N}\left(a^{*}(f) a(g)\right)=(g, N f)$ and Wick's lemma. Let $\left(\mathcal{H}_{N}, \pi_{N}, \Omega_{N}\right)$ be the cyclic (or GNS) representation of $\omega_{N}$ :

$$
\omega_{N}(A)=\left(\Omega_{N}, \pi_{N}(A) \Omega_{N}\right), \quad(A \in \mathcal{A}(\mathcal{H})) .
$$

The algebra of observables is the (strong) closure of the range of $\pi_{N}$, which is equal to its double commutant $\overline{\pi_{N}(\mathcal{A}(\mathcal{H}))}=\pi_{N}(\mathcal{A}(\mathcal{H}))^{\prime \prime}$. We also recall that a state is pure if and only if $\pi_{N}(\mathcal{A}(\mathcal{H}))$ is irreducible, i.e. $\pi_{N}(\mathcal{A}(\mathcal{H}))^{\prime}=\{c \cdot 1 \mid c \in \mathbb{C}\}$, see e.g. [5], Thm. 2.3.19. This is equivalent to $N$ being a projection operator.

These concepts briefly reviewed, we are now ready to state our main theorem. Its significance is discussed below in a series of remarks. The key result, which is part (v) together with Corollary 2, states that the moment generating function is given by the regularized determinant, as described in the previous section.

Theorem 1. Assume that

$$
\begin{aligned}
& {[Q, N]=0,} \\
& \sqrt{N}-\sqrt{N_{U^{*}}}, \quad \sqrt{N^{\prime}}-\sqrt{N_{U^{*}}^{\prime}} \in \mathcal{I}_{1},
\end{aligned}
$$

where $N_{U^{*}}=U N U^{*}$.

Pure state. Suppose $N=N^{2}$. Then we have

i. $\widetilde{D}(\lambda)-1 \in \mathcal{I}_{1}$, where $\widetilde{D}(\lambda)$ is given in Eq. (9).

ii. The Bogoliubov automorphisms induced on $\mathcal{A}(\mathcal{H})$ by the unitary operators $U$ and $\exp (\mathrm{i} \lambda Q)$ are implementable on $\mathcal{H}_{N}$ : There exist a unitary operator $\widehat{U}$ and $a$ selfadjoint $\widehat{Q}$ on $\mathcal{H}_{N}$ such that

$$
\begin{aligned}
& \widehat{U} \pi_{N}\left(a^{\#}(f)\right) \widehat{U}^{*}=\pi_{N}\left(a^{\#}(U f)\right), \\
& \mathrm{e}^{\mathrm{i} \lambda \widehat{Q}} \pi_{N}\left(a^{\#}(f)\right) \mathrm{e}^{-\mathrm{i} \lambda \widehat{Q}}=\pi_{N}\left(a^{\#}\left(\mathrm{e}^{\mathrm{i} \lambda Q} f\right)\right),
\end{aligned}
$$

for all $f \in \mathcal{H}$.

iii. $\mathrm{e}^{\mathrm{i} \lambda \widehat{Q}} \in \pi_{N}(\mathcal{A}(\mathcal{H}))^{\prime \prime}$. More generally, $f(\widehat{Q}) \in \pi_{N}(\mathcal{A}(\mathcal{H}))^{\prime \prime}$ for any bounded function $f$.

iv. The above properties define $\widehat{U}$ uniquely up to left multiplication with an element from $\pi_{N}(\mathcal{A}(\mathcal{H}))^{\prime}$, and $\widehat{Q}$ up to an additive constant. In particular, $\widehat{U}^{*} \mathrm{e}^{\mathrm{i} \lambda \widehat{Q}} \widehat{U} \mathrm{e}^{-\mathrm{i} \lambda \widehat{Q}}$ is unaffected by the ambiguities.

v.

$$
\left(\Omega_{N}, \widehat{U}^{*} \mathrm{e}^{\mathrm{i} \lambda \widehat{Q}} \widehat{U} \mathrm{e}^{-\mathrm{i} \lambda \widehat{Q}} \Omega_{N}\right)=\operatorname{det} \widetilde{D}(\lambda) .
$$

Mixed state. The above conclusions hold also for $0<N<1$ if, in addition,

$$
Q \sqrt{N N^{\prime}} \in \mathcal{I}_{1}
$$

Remark. 1) Equation (15) demands that the evolution $U$ preserves $N$, except for creating excitations within an essentially finite region in space and energy, as can be seen from the phase space picture given in the introduction. This assumption is appropriate for the evolution induced by a compact device operating smoothly during a finite time interval.

2) The operators $\widehat{U}, \widehat{Q}$ in (ii) are replacements for the non-existent $\Gamma(U)$ and $\mathrm{d} \Gamma(Q)$ mentioned in the introduction. Equations $(16,17)$ state that any additional particle in the system evolves by $U$, resp. contributes to the charge as described by $Q$. 
3) If the state is pure, the pair of Eqs. (15) reduce to the first one with square roots dropped, and property (iii) holds trivially, since $\mathcal{B}\left(\mathcal{H}_{N}\right)=\pi_{N}(\mathcal{A}(\mathcal{H}))^{\prime \prime}$, the bounded operators on $\mathcal{H}_{N}$. Moreover, $\widehat{U}$ is unique up to a phase. Incidentally, condition (19) would be trivial in this case.

4) Property (iii) states that $\widehat{Q}$ is an observable, and the same is true for $\widehat{U}^{*} \widehat{Q} \widehat{U}$, because of $\widehat{U}^{*} \pi_{N}(\mathcal{A}(\mathcal{H})) \widehat{U} \subset \pi_{N}(\mathcal{A}(\mathcal{H}))$, see (16). Thus, the total charges before and after the evolution are separately bestowed with physical meaning.

5) The physical origin of the extra assumption (19) needed in the mixed state case is as follows. In both cases, the expected charge contained in a portion of the lead is of order of its length $L$, or zero if renormalized by subtraction of a background charge. In the pure case however, the Fermi sea is an eigenvector of the charge operator, while for the mixed state, the variance of the charge must itself be of order $L$, because the occupation of the one-particle states is fluctuating, since $N N^{\prime} \neq 0$. Hence, in this latter situation, the measurement of the renormalized charge yields finite values only as long as $L$ is finite, of which Eq. (19) is a mathematical abstraction. This condition, while unnecessary for property (ii), is essential for (iii). Without the latter, the 1.h.s. of Eq. (18) appears to be ambiguous. On the other hand, the weaker condition

$$
\left(Q_{U}-Q\right) \sqrt{N N^{\prime}} \in \mathcal{I}_{1}
$$

is sufficient for property (i) and to ensure that the difference $\widehat{Q_{U}-} Q$ is an observable.

6) The theorem does not apply to the general case (11). The two cases considered suffice for thermal states with $\beta=\infty$ and $0<\beta<\infty$.

Let $\widehat{Q}=\int n d P(n)$ be the spectral representation of $\widehat{Q}$. According to quantum mechanical principles an ideal measurement of $\widehat{Q}$ with outcome $n$ in $d n$ collapses $\Omega_{N}$ to the state $d P(n) \Omega_{N}$, normalized to the probability $\left(d P(n) \Omega_{N}, d P(n) \Omega_{N}\right)$ of that outcome. Effectively, this means that $d P(n) \Omega_{N}$ is the state relevant for a second measurement. The charge transfer is inferred from two measurements [15] of the charge $\widehat{Q}$, one before and one after the evolution of the system by $\widehat{U}$. The joint probability for measurements $n$ and $m$ is $\left(\widehat{U} d P(n) \Omega_{N}, d P(m) \widehat{U} d P(n) \Omega_{N}\right)$ and the generating function appropriately defined as

$$
\chi_{N}(\lambda)=\iint\left(d P(n) \Omega_{N}, \widehat{U}^{*} d P(m) \widehat{U} d P(n) \Omega_{N}\right) \mathrm{e}^{\mathrm{i} \lambda(m-n)} .
$$

Corollary 2. The spectrum of $\widehat{Q}$ consists of integers, up to an additive constant. The generating function is

$$
\chi_{N}(\lambda)=\left(\Omega_{N}, \widehat{U}^{*} \mathrm{e}^{\mathrm{i} \lambda \widehat{Q}} \widehat{U} \mathrm{e}^{-\mathrm{i} \lambda \widehat{Q}} \Omega_{N}\right)
$$

and describes the transport of integer charges $n$ with non-negative probabilities:

$$
\chi_{N}(\lambda)=\sum_{n \in \mathbb{Z}} p_{n} \mathrm{e}^{\mathrm{i} \lambda n}, \quad p_{n} \geq 0, \quad \sum_{n \in \mathbb{Z}} p_{n}=1 .
$$

Moreover, the particle-hole symmetry (10) holds true. 


\section{Proofs}

We begin by giving the proof of the corollary and continue with that of part (i) of the theorem. Then we give some preliminaries, including details such as inner Bogoliubov automorphisms and the Shale-Stinespring criterion for general ones. Thereafter we prove parts (ii-iv) readily if the state is pure, and using its purification, if it is mixed. Finally, the main formula (v) is obtained using an approximation procedure in terms of inner automorphisms and finite dimensional determinants.

4.1. Proof of Corollary 2. We begin by recalling that every gauge-invariant state is a factor state (see [17], Thm. 5.1), i.e.

$$
\pi_{N}(\mathcal{A}(\mathcal{H}))^{\prime} \cap \pi_{N}(\mathcal{A}(\mathcal{H}))^{\prime \prime}=\{c \cdot 1 \mid c \in \mathbb{C}\} .
$$

From Eq. (17) and $\mathrm{e}^{2 \pi \mathrm{i} Q}=1$, we see that $\mathrm{e}^{2 \pi \mathrm{i} \widehat{Q}} \in \pi_{N}(\mathcal{A}(\mathcal{H}))^{\prime}$, while by (iii) we have $\mathrm{e}^{2 \pi \mathrm{i} \widehat{Q}} \in \pi_{N}(\mathcal{A}(\mathcal{H}))^{\prime \prime}$. Thus $\mathrm{e}^{2 \pi \mathrm{i} \widehat{Q}}=c,(|c|=1)$ and we may assume $c=1$ by adding an additive constant to $\widehat{Q}$, see (iv). The spectral representation of $\widehat{Q}$ is then of the form

$$
\widehat{Q}=\sum_{n \in \mathbb{Z}} n P_{n} .
$$

We note that

$$
\left(\Omega_{N}, \mathrm{e}^{\mathrm{i} \lambda \widehat{Q}} A \mathrm{e}^{-\mathrm{i} \lambda \widehat{Q}} \Omega_{N}\right)=\left(\Omega_{N}, A \Omega_{N}\right)
$$

for $A \in \pi_{N}(\mathcal{A}(\mathcal{H}))^{\prime \prime}$. Indeed, for $A=\pi_{N}\left(a^{*}(f) a(g)\right)$, we have $\mathrm{e}^{\mathrm{i} \lambda \widehat{Q}} A \mathrm{e}^{-\mathrm{i} \lambda \widehat{Q}}=$ $\pi_{N}\left(a^{*}\left(\mathrm{e}^{\mathrm{i} \lambda Q} f\right) a\left(\mathrm{e}^{\mathrm{i} \lambda Q_{g}}\right)\right)$ by (17). The expectations (23) agree because of $\left(\mathrm{e}^{\mathrm{i} \lambda Q_{g}, N}\right.$ $\left.\mathrm{e}^{\mathrm{i} \lambda Q} f\right)=(g, N f)$ by $[Q, N]=0$. The same holds true by (12) for arbitrary products of $a^{*}\left(f_{i}\right), a\left(g_{i}\right)$, and by density, for $A \in \pi_{N}(\mathcal{A}(\mathcal{H}))^{\prime \prime}$. By (iii) we may apply (23) to $A \mathrm{e}^{\mathrm{i} \lambda \widehat{Q}}$ instead of $A$, and obtain $\left(\Omega_{N}, P_{n} A \Omega_{N}\right)=\left(\Omega_{N}, A P_{n} \Omega_{N}\right)$; then this to $A P_{n} \in \pi_{N}(\mathcal{A}(\mathcal{H}))^{\prime \prime}$ instead of $A$, and get $\left(\Omega_{N}, A P_{n} \Omega_{N}\right)=\left(\Omega_{N}, P_{n} A P_{n} \Omega_{N}\right)$. Moreover, we have $\widehat{U}^{*} \mathrm{e}^{\mathrm{i} \lambda \widehat{Q}} \widehat{U} \in \widehat{U}^{*} \pi_{N}(\mathcal{A}(\mathcal{H}))^{\prime \prime} \widehat{U} \subset \pi_{N}(\mathcal{A}(\mathcal{H}))^{\prime \prime}$ by (16). Hence, using (22), we see that

$$
\begin{aligned}
& \left(\Omega_{N}, \widehat{U}^{*} \mathrm{e}^{\mathrm{i} \lambda \widehat{Q}} \widehat{U} \mathrm{e}^{-\mathrm{i} \lambda \widehat{Q}} \Omega_{N}\right)=\sum_{n \in \mathbb{Z}}\left(\Omega_{N}, P_{n} \widehat{U}^{*} \mathrm{e}^{\mathrm{i} \lambda \widehat{Q}} \widehat{U} P_{n} \Omega_{N}\right) \mathrm{e}^{-\mathrm{i} \lambda n} \\
& =\sum_{n, m \in \mathbb{Z}}\left(\Omega_{N}, P_{n} \widehat{U}^{*} P_{m} \widehat{U} P_{n} \Omega_{N}\right) \mathrm{e}^{\mathrm{i} \lambda(m-n)}
\end{aligned}
$$

is of the stated form.

4.2. Part (i). Since the projection $Q$ commutes with $N$, see (14), we have

$$
\begin{aligned}
\mathrm{e}^{\mathrm{i} \lambda N Q} & =1+\left(\mathrm{e}^{\mathrm{i} \lambda N}-1\right) Q, \\
\mathrm{e}^{-\mathrm{i} \lambda N_{U} Q_{U}} & =1+Q_{U}\left(\mathrm{e}^{-\mathrm{i} \lambda N_{U}}-1\right) .
\end{aligned}
$$

We insert these equations in the definition (9) of $\widetilde{D}(\lambda)$. Moreover,

$$
N_{U}-N=N^{1 / 2}\left(N_{U}^{1 / 2}-N^{1 / 2}\right)+\left(N_{U}^{1 / 2}-N^{1 / 2}\right) N_{U}^{1 / 2} \in \mathcal{I}_{1},
$$


so that

$$
\mathrm{e}^{-\mathrm{i} \lambda N}-\mathrm{e}^{-\mathrm{i} \lambda N_{U}}=\mathrm{i} \int_{0}^{\lambda} \mathrm{e}^{-\mathrm{i}(\lambda-s) N_{U}}\left(N_{U}-N\right) \mathrm{e}^{-\mathrm{i} s N} \mathrm{~d} s
$$

also belongs to the trace class ideal. Rather than proving $\widetilde{D}(\lambda) \in 1+\mathcal{I}_{1}$ for $\widetilde{D}(\lambda)$ we may thus do so for the expression

$$
\begin{aligned}
{[1+} & \left.Q_{U}\left(\mathrm{e}^{-\mathrm{i} \lambda N}-1\right)\right] N^{\prime}\left[1+\left(\mathrm{e}^{\mathrm{i} \lambda N}-1\right) Q\right]+\left[1+Q_{U}\left(\mathrm{e}^{\mathrm{i} \lambda N^{\prime}}-1\right)\right] N\left[1+\left(\mathrm{e}^{-\mathrm{i} \lambda N^{\prime}}-1\right) Q\right] \\
= & N^{\prime}+N+Q_{U}\left[\left(\mathrm{e}^{-\mathrm{i} \lambda N}-1\right) N^{\prime}+\left(\mathrm{e}^{\mathrm{i} \lambda N^{\prime}}-1\right) N\right]+\left[N^{\prime}\left(\mathrm{e}^{\mathrm{i} \lambda N}-1\right)+N\left(\mathrm{e}^{-\mathrm{i} \lambda N^{\prime}}-1\right)\right] Q \\
& +Q_{U}\left[\left(\mathrm{e}^{-\mathrm{i} \lambda N}-1\right) N^{\prime}\left(\mathrm{e}^{\mathrm{i} \lambda N}-1\right)+\left(\mathrm{e}^{\mathrm{i} \lambda N^{\prime}}-1\right) N\left(\mathrm{e}^{-\mathrm{i} \lambda N^{\prime}}-1\right)\right] Q \\
= & 1+Q_{U}\left[(\cos (\lambda N)-1) N^{\prime}+\left(\cos \left(\lambda N^{\prime}\right)-1\right) N-\mathrm{i} \sin (\lambda N) N^{\prime}+\mathrm{i} \sin \left(\lambda N^{\prime}\right) N\right] \\
& +Q\left[(\cos (\lambda N)-1) N^{\prime}+\left(\cos \left(\lambda N^{\prime}\right)-1\right) N+\mathrm{i} \sin (\lambda N) N^{\prime}-\mathrm{i} \sin \left(\lambda N^{\prime}\right) N\right] \\
& +2 Q_{U} Q\left[(1-\cos (\lambda N)) N^{\prime}+\left(1-\cos \left(\lambda N^{\prime}\right)\right) N\right] \\
= & 1+\left(Q_{U}^{2}+Q^{2}-2 Q_{U} Q\right)\left[(\cos (\lambda N)-1) N^{\prime}+\left(\cos \left(\lambda N^{\prime}\right)-1\right) N\right] \\
& +\mathrm{i}\left(Q-Q_{U}\right)\left[\sin (\lambda N) N^{\prime}-\sin \left(\lambda N^{\prime}\right) N\right] .
\end{aligned}
$$

With the help of the functions $f(x)=(\cos x-1) / x$ and $g(x)=(\sin x) / x$, which are bounded also at $x=0$, the expression is rewritten as

$$
\begin{aligned}
& 1+\left[\left(Q-Q_{U}\right) Q-Q_{U}\left(Q-Q_{U}\right)\right] N N^{\prime} \lambda\left(f(\lambda N)+f\left(\lambda N^{\prime}\right)\right) \\
& \quad+\mathrm{i}\left(Q-Q_{U}\right) N N^{\prime} \lambda\left(g(\lambda N)-g\left(\lambda N^{\prime}\right)\right) .
\end{aligned}
$$

Besides $Q \sqrt{N N^{\prime}} \in \mathcal{I}_{1}$, see Eq. (19), we have $Q_{U} \sqrt{N N^{\prime}}=U^{*} Q \sqrt{N_{U^{*} N_{U^{*}}^{\prime}}} U \in \mathcal{I}_{1}$ by Eq. (15), and hence $\left(Q-Q_{U}\right) \sqrt{N N^{\prime}} \in \mathcal{I}_{1}$, cf. (20). This makes the claim manifest.

In the zero temperature case, where $N$ is a projection, the above proof simplifies considerably due to $N N^{\prime}=0$.

4.3. Preliminaries. We recall a few results about Bogoliubov transformations, first inner and then others.

Given a bounded operator $A$ on $\mathcal{H}$, operators $\Gamma(A)$ and $\mathrm{d} \Gamma(A)$ are usually defined on the Fock space over $\mathcal{H}$. Following [2] we define them instead as elements of the CAR-algebra $\mathcal{A}(\mathcal{H})$, when $A$ is of finite rank.

- $\quad$ For rank one operators $A_{i}=\left|f_{i}\right\rangle\left\langle g_{i}\right|,(i=1, \ldots, n)$, we set

$$
\mathrm{d} \Gamma\left(A_{1}, \ldots, A_{n}\right)=a^{*}\left(f_{n}\right) \cdots a^{*}\left(f_{1}\right) a\left(g_{1}\right) \cdots a\left(g_{n}\right) .
$$

The definition is extended by multilinearity to operators $A_{i}$ of finite rank. The result is independent of the particular decomposition into rank one operators.

- For $U-1$ of finite rank, we set

$$
\Gamma(U)=\sum_{n=0}^{\infty} \frac{1}{n !} \mathrm{d} \Gamma(\underbrace{U-1, \ldots, U-1}_{n}),
$$

where the term $n=0$, in which no arguments are present, is read as $\mathrm{d} \Gamma=1$. The sum is finite, because the terms with $n>\operatorname{rank}(U-1)$ vanish. 
The elements of $\mathcal{A}(\mathcal{H})$ just defined share the properties of the operators on Fock space known by the same notation.

Lemma 3. Let $U-1$ be of finite rank. Then

$$
\begin{aligned}
\Gamma(U) a^{*}(f) & =a^{*}(U f) \Gamma(U), \\
\Gamma\left(U_{1} U_{2}\right) & =\Gamma\left(U_{1}\right) \Gamma\left(U_{2}\right) .
\end{aligned}
$$

In particular, $\Gamma(U)$ is unitary if $U$ is.

Proof. We have

$$
\begin{aligned}
\mathrm{d} \Gamma\left(A_{1}, \ldots, A_{n}\right) a^{*}(f)= & a^{*}(f) \mathrm{d} \Gamma\left(A_{1}, \ldots, A_{n}\right) \\
& +\sum_{i=1}^{n} a^{*}\left(A_{i} f\right) \mathrm{d} \Gamma\left(A_{1}, \ldots, \widehat{A}_{i}, \ldots, A_{n}\right),
\end{aligned}
$$

where the hat indicates omission. In the rank one case, $A_{i}=\left|f_{i}\right\rangle\left\langle g_{i}\right|$, this follows from (25) and from $\left(g_{i}, f\right) a^{*}\left(f_{i}\right)=a^{*}\left(A_{i} f\right)$. In the general case, by multilinearity. Thus,

$$
\begin{aligned}
\Gamma(U) a^{*}(f) & =a^{*}(f) \Gamma(U)+a^{*}((U-1) f) \sum_{n=1}^{\infty} \frac{1}{(n-1) !} \mathrm{d} \Gamma(U-1, \ldots, U-1) \\
& =a^{*}(f) \Gamma(U)+a^{*}((U-1) f) \Gamma(U)=a^{*}(U f) \Gamma(U),
\end{aligned}
$$

since we applied (28) with $n$ equal entries $A_{i}=U-1$.

We have

$$
\begin{aligned}
& \mathrm{d} \Gamma\left(A_{1}, \ldots, A_{n}\right) \mathrm{d} \Gamma\left(B_{1}, \ldots, B_{m}\right) \\
& =\sum_{l=0}^{\min (n, m)} \sum_{\mathcal{C}_{l}} \mathrm{~d} \Gamma\left(A_{i_{1}} B_{j_{1}}, \ldots, A_{i_{l}} B_{j_{l}}, A_{1}, \ldots, \widehat{A}_{i_{s}}, \ldots, A_{n}, B_{1}, \ldots, \widehat{B}_{j_{s}}, \ldots, B_{m}\right),
\end{aligned}
$$

where the second sum runs over all $l$-contractions $\left(i_{1}, j_{1}\right), \ldots,\left(i_{l}, j_{l}\right)$ with $i_{1}<\ldots<$ $i_{l}, j_{i_{s}} \neq j_{i_{r}}$. In the rank one case, which implies the general one, this is just Wick's lemma for normal ordered products. Thus

$$
\begin{aligned}
\Gamma\left(U_{1}\right) \Gamma\left(U_{2}\right)= & \sum_{n=0}^{\infty} \frac{1}{n !} \mathrm{d} \Gamma\left(U_{1}-1, \ldots, U_{1}-1\right) \cdot \sum_{m=0}^{\infty} \frac{1}{m !} \mathrm{d} \Gamma\left(U_{2}-1, \ldots, U_{2}-1\right) \\
= & \sum_{n, m=0}^{\infty} \sum_{l=0}^{\min (n, m)} \frac{1}{l !(n-l) !(m-l) !} \mathrm{d} \Gamma\left(\left(U_{1}-1\right)\left(U_{2}-1\right), \ldots,\right. \\
& \left.\times U_{1}-1, \ldots, U_{2}-1, \ldots\right)
\end{aligned}
$$

with entries repeated $l, n-l, m-l$ times. In fact, the number of $l$-contractions is

$$
\frac{1}{l !} \frac{n !}{(n-l) !} \frac{m !}{(m-l) !} .
$$


Setting $n-l=: s, m-l=: t, l+s+t=: r$, we have

$$
\begin{aligned}
\Gamma\left(U_{1}\right) \Gamma\left(U_{2}\right) & =\sum_{r=0}^{\infty} \sum_{\substack{l, s, t \\
l+s+t=r}} \frac{1}{l ! s ! t !} \mathrm{d} \Gamma\left(\left(U_{1}-1\right)\left(U_{2}-1\right), \ldots, U_{1}-1, \ldots, U_{2}-1, \ldots\right) \\
& =\sum_{r=0}^{\infty} \frac{1}{r !} \mathrm{d} \Gamma\left(\left(U_{1}-1\right)\left(U_{2}-1\right)+\left(U_{1}-1\right)+\left(U_{2}-1\right), \ldots\right),
\end{aligned}
$$

since there are $r ! / l ! s ! t$ ! ways to pick terms from each entry of the last line. Since $\left(U_{1}-1\right)\left(U_{2}-1\right)+\left(U_{1}-1\right)+\left(U_{2}-1\right)=U_{1} U_{2}-1$, the proof is complete.

If $O$ is an operator on $\mathcal{H}$ such that $O-1$ is in the trace class, its Fredholm determinant is defined by

$$
\operatorname{det} O=\sum_{k=0}^{\infty} \operatorname{tr} \wedge^{k}(O-1)
$$

This extends the usual definition of the determinant in the finite dimensional case.

Lemma 4. Let $A$ be a finite rank operator, and $0 \leq N \leq 1$. Then

$$
\omega_{N}(\mathrm{~d} \Gamma(\underbrace{A, \ldots, A}_{k}))=\operatorname{tr} \wedge^{k}(A N) .
$$

Moreover, if $U$ is such that $U-1$ is of finite rank, then

$$
\omega_{N}(\Gamma(U))=\operatorname{det}((1-N)+U N) .
$$

Proof. The trace of a finite rank operator $A=\sum_{i=1}^{m} f_{i}\left(g_{i}, \cdot\right)$ is $\operatorname{tr} A=\sum_{i=1}^{m}\left(g_{i}, f_{i}\right)$. By the same token, that of

$$
\wedge^{k} A=\sum_{i_{1}, \ldots i_{k}=1}^{m} \frac{1}{k !} \sum_{\sigma \in S_{k}}(-1)^{\sigma} \otimes_{\alpha=1}^{k} f_{i_{\sigma(\alpha)}}\left(g_{i_{\alpha}}, \cdot\right)
$$

is

$$
\operatorname{tr} \wedge^{k} A=\sum_{1 \leq i_{1}<\ldots<i_{k} \leq m} \operatorname{det}\left(g_{i_{\alpha}}, f_{i_{\beta}}\right)_{\alpha, \beta=1}^{k} .
$$

Since the $a^{\#}(f)$ anticommute, we have

$$
\mathrm{d} \Gamma(A, \ldots, A)=\sum_{1 \leq i_{1}<\ldots<i_{k} \leq m} a^{*}\left(f_{i_{1}}\right) \cdots a^{*}\left(f_{i_{k}}\right) a\left(g_{i_{k}}\right) \cdots a\left(g_{i_{1}}\right),
$$

whose expectation value is computed by (12) as

$$
\omega_{N}(\mathrm{~d} \Gamma(A, \ldots, A))=\sum_{1 \leq i_{1}<\ldots<i_{k} \leq m} \operatorname{det}\left(N g_{i_{\alpha}}, f_{i_{\beta}}\right)_{\alpha, \beta=1}^{k}=\operatorname{tr} \wedge^{k}(A N),
$$

because the decomposition of $A N$ differs from that of $A$ by $N g_{i}$ in place of $g_{i}$. This proves the first part of the lemma. The second part is now an application of the definition of the determinant, Eq. (29). Indeed, $\omega_{N}(\Gamma(U))=1+\sum_{k=1}^{\infty} \operatorname{tr} \wedge^{k}((U-1) N)=$ $\operatorname{det}(1+(U-1) N)$. 
We recall a few results on Bogoliubov transformations. Their proofs can be found e.g. in [1], where however CAR-algebras are introduced in the self-dual guise. A remark at the end of this subsection is intended as an aid to translation. The first result is the Shale-Stinespring criterion [24] about unitary implementability (see e.g. [14,1], Thm. $6.3(1))$.

Proposition 5. Let $P$ be a projection and $V$ a unitary operator on $\mathcal{H}$. The Bogoliubov automorphism induced by $V$ on $\mathcal{H}$, i.e. $a(f) \mapsto a(V f)$, is unitarily implementable in the representation $\pi_{P}$ if and only if $P V(1-P)$ and $(1-P) V P$ are in the Hilbert-Schmidt class, $\mathcal{I}_{2}$.

In particular, an equivalent condition is $[P, V]=P V(1-P)-(1-P) V P \in \mathcal{I}_{2}$. There is a version of this proposition for groups ([1], Thm. $6.10(2,3))$.

Proposition 6. Let $V$ in the previous proposition be replaced by a 1-parameter unitary group $V_{\lambda},(\lambda \in \mathbb{R})$, such that $V_{\lambda}$ is norm continuous and $P V_{\lambda}(1-P)$ is continuous in the $\mathcal{I}_{2}$-norm. Then $V_{\lambda}$ has an implementer of the form $\widehat{V}_{\lambda}=\exp (\mathrm{i} \lambda \widehat{v})$, where $\widehat{v}$ is a self-adjoint operator on $\mathcal{H}_{P}$. The requirement

$$
\left(\Omega_{P}, \widehat{v} \Omega_{P}\right)=0
$$

may be imposed, in which case $\widehat{v}$ is unique.

An equivalent condition, is $V_{\lambda}=\exp (\mathrm{i} \lambda v)$ with $v$ a bounded, selfadjoint operator on $\mathcal{H}$, and $P v(1-P) \in \mathcal{I}_{2}$.

The next result is about the continuity of the implementation, see [1], Thm. 6.10 (7).

Proposition 7. Let $v$ and $v_{n},(n=0,1, \ldots)$, satisfy the hypotheses of Prop. 6, and let $\widehat{v}$, $\widehat{v_{n}}$ satisfy the normalization (31). If $\left(v_{n}\right)$ converge strongly to $v$ and if $\| P\left(v_{n}-v\right)(1-$ $P) \|_{2} \rightarrow 0$ as $n \rightarrow \infty$, then

$$
\mathrm{s}-\lim _{n} \mathrm{e}^{\mathrm{i} \lambda \widehat{v_{n}}}=\mathrm{e}^{\mathrm{i} \lambda \widehat{v}}
$$

The last preliminary is concerned with the twisted duality of CAR-algebras. Let $P$ be an orthogonal projection on $\mathcal{H}, \mathcal{K} \subset \mathcal{H}$ a closed subspace, and $\mathcal{K}^{\perp}$ its orthogonal complement. Let $\widetilde{\mathcal{A}}\left(\mathcal{K}^{\perp}\right)$ be the von Neumann algebra generated by $\widehat{\Lambda} \pi_{P}(a(f)),(f \in$ $\left.\mathcal{K}^{\perp}\right)$, where $\widehat{\Lambda}$ is the parity. Then $[7,4]$

$$
\pi_{P}(\mathcal{A}(\mathcal{K}))^{\prime}=\widetilde{\mathcal{A}}\left(\mathcal{K}^{\perp}\right) .
$$

The implementers $\widehat{V}$ from Prop. 5 commute with parity: Let $\widehat{\Lambda}: \mathcal{H}_{P} \rightarrow \mathcal{H}_{P}$ be the unitary implementation of the *-automorphism $a(f) \mapsto a(-f)$ which is uniquely determined by $\widehat{\Lambda} \Omega_{P}=\Omega_{P}$. Then

$$
[\widehat{V}, \widehat{\Lambda}]=0,
$$

see [1], Thm. 6.3 (3), Thm. 6.7 (2). We will actually apply this fact only to $\widehat{V}=\widehat{V}_{\lambda}$ as in Prop. 6, in which case it can be verified as follows. Since $\left[V_{\lambda},-1\right]=0$, the operators $\widehat{\Lambda} \widehat{V}_{\lambda}$ and $\widehat{V}_{\lambda} \widehat{\Lambda}$ implement the same Bogoliubov automorphism, whence $\widehat{\Lambda} \widehat{V}_{\lambda}=c_{\lambda} \widehat{V}_{\lambda} \widehat{\Lambda}$ with $\left|c_{\lambda}\right|=1$. From $\left(\Omega_{P}, \widehat{V}_{\lambda} \Omega_{P}\right)=\left(\widehat{\Lambda} \Omega_{P}, \widehat{\Lambda} \widehat{V}_{\lambda} \Omega_{P}\right)=c_{\lambda}\left(\Omega_{P}, \widehat{V}_{\lambda} \Omega_{P}\right)$ we find $c_{\lambda}=1$ for small $\lambda$, because $\left(\Omega_{P}, \widehat{V}_{\lambda} \Omega_{P}\right) \rightarrow 1,(\lambda \rightarrow 0)$. The conclusion extends to all $\lambda$ by the group property. 
Remark. In order to make contact with the repeatedly cited article [1] we recall that a self-dual CAR-algebra $\mathcal{A}(\widetilde{\mathcal{H}}, \Gamma)$ is given in terms of a separable Hilbert space $\widetilde{\mathcal{H}}$ equipped with a conjugation $\Gamma$. Its generators $B(h)$ are linear in $h \in \widetilde{\mathcal{H}}$ and the relations are $B(h)^{*}=B(\Gamma h)$ and $\left\{B(h), B\left(h^{\prime}\right)^{*}\right\}=\left(h^{\prime}, h\right) 1$. A projection $\widetilde{P}$ on $\widetilde{\mathcal{H}}$ satisfying $\widetilde{P}+\Gamma \widetilde{P} \Gamma=1$ defines a pure state $\omega$ on the algebra through

$$
\omega\left(B(h)^{*} B(h)\right)=0, \quad(\widetilde{P} h=0) .
$$

The algebra $\mathcal{A}(\mathcal{H})$ is connected to the above by picking a conjugation $C$ on $\mathcal{H}$ and by setting

$$
\widetilde{\mathcal{H}}=\mathcal{H} \oplus \mathcal{H}, \quad \Gamma(f \oplus g)=C g \oplus C f, \quad B(f \oplus g)=a^{*}(f)+a(C g) .
$$

States defined by $P$ and $\widetilde{P}$ then agree if $\widetilde{P}(f \oplus g)=(1-P) f \oplus C P C g$.

4.4. Parts (ii-iv). Pure state, $N=N^{2}$. Existence: In the case $V_{\lambda}=\exp (\mathrm{i} \lambda Q)$ we have $\left[N, V_{\lambda}\right]=0$ by Eq. (14), so that existence of a unitary implementer $\widehat{V}_{\lambda}=\exp (\mathrm{i} \lambda \widehat{Q})$ is trivial by Prop. 6. Similarly, in the case $V=U$ we have $[N, U]=\left(N-N_{U^{*}}\right) U \in \mathcal{I}_{2}$ by Eq. (24). Hence it is also implementable by Prop. 5 .

Uniqueness: Let $\widehat{V}$ denote either $\exp (\mathrm{i} \lambda \widehat{Q})$ or $\widehat{U}$. Suppose $\widehat{V}_{1}$ and $\widehat{V}_{2}$ both implement the same transformation. Then $\widehat{V}_{1} \widehat{V}_{2}^{*} \pi_{N}(a(V f))=\pi_{N}(a(V f)) \widehat{V}_{1} \widehat{V}_{2}^{*}$. Thus $\widehat{V}_{1}=$ $\left(\widehat{V}_{1} \widehat{V}_{2}^{*}\right) \widehat{V}_{2}$ and $\widehat{V}_{2}$ differ by left multiplication with $\widehat{V}_{1} \widehat{V}_{2}^{*} \in \pi_{N}(\mathcal{A}(\mathcal{H}))^{\prime}$. In the pure case the cyclic representation is irreducible, whence $\widehat{U}$ is unique up to a phase and $\widehat{Q}$ up to an additive constant. As mentioned in Remark 3, property (iii) is empty in this case. Mixed state, $0<N<1$. Given $0 \leq N \leq 1$ on $\mathcal{H}$, we consider its purification

$$
P_{N}=\left(\begin{array}{cc}
N & \sqrt{N N^{\prime}} \\
\sqrt{N N^{\prime}} & N^{\prime}
\end{array}\right)=P_{N}^{2}
$$

on $\mathcal{H} \oplus \mathcal{H}$, together with the cyclic representation $\left(\mathcal{H}_{P_{N}}, \pi_{P_{N}}, \Omega_{P_{N}}\right)$ of the state defined by $P_{N}$ on $\mathcal{A}(\mathcal{H} \oplus \mathcal{H})$. We can identify

$$
\mathcal{A}(\mathcal{H}) \cong \mathcal{A}(\mathcal{H} \oplus 0)
$$

$\operatorname{via} a(f)=a(f \oplus 0)$, and

$$
\mathcal{H}_{N} \equiv \pi_{P_{N}}(\mathcal{A}(\mathcal{H} \oplus 0))^{\prime \prime} \Omega_{P_{N}} \subset \mathcal{H}_{P_{N}}, \quad \Omega_{N} \equiv \Omega_{P_{N}}, \quad \pi_{N}(a) \equiv \pi_{P_{N}}(a)\left\lceil\mathcal{H}_{N},\right.
$$

since these objects satisfy

$$
\left(\Omega_{P_{N}}, \pi_{P_{N}}\left(a^{*}(f \oplus 0) a(g \oplus 0)\right) \Omega_{P_{N}}\right)=\left(g \oplus 0, P_{N}(f \oplus 0)\right)=(g, N f),
$$

as required by (13). We can not handle the most general mixed case $0 \leq N \leq 1$. The reason comes from the following lemma, whose proof is postponed till the end of the section.

Lemma 8. Assume $0<N<1$ (strict inequality). Then $\Omega_{P_{N}}$ is cyclic in $\mathcal{H}_{P_{N}}$ for $\pi_{P_{N}}(\mathcal{A}(\mathcal{H} \oplus 0))$. In particular, we have equality in (34), $\mathcal{H}_{N}=\mathcal{H}_{P_{N}}$. 
A unitary $V$ on $\mathcal{H}$ induces two automorphisms on $\mathcal{A}(\mathcal{H} \oplus \mathcal{H})$ : (a) $a(f \oplus g) \mapsto$ $a(V f \oplus g)$, and (b) $a(f \oplus g) \mapsto a(V f \oplus V g)$, whose implementation may be envisaged:

(a) $\widehat{V} \pi_{P_{N}}(a(f \oplus g)) \widehat{V}^{*}=\pi_{P_{N}}(a(V f \oplus g))$,
(b) $\widehat{V} \pi_{P_{N}}(a(f \oplus g)) \widehat{V}^{*}=\pi_{P_{N}}(a(V f \oplus V g))$.

Both choices for $\widehat{V}$ would provide an implementation for $V$ in the representation $\pi_{N}$ on $\mathcal{H}_{N}$. Only in the first case we have $\widehat{V} \in \pi_{N}(\mathcal{A}(\mathcal{H}))^{\prime \prime}$. Indeed, by (32) applied to $\mathcal{H} \oplus 0 \subset \mathcal{H} \oplus \mathcal{H}$ instead of $\mathcal{K} \subset \mathcal{H}$, we need to check $\widehat{V} \in \widetilde{\mathcal{A}}(0 \oplus \mathcal{H})^{\prime}$. This however follows from $\left[\widehat{V}, \pi_{P_{N}}(a(0 \oplus g))\right]=0$, see $(36)$, and from $[\widehat{V}, \widehat{\Lambda}]=0$, see $(33)$.

In order to determine the existence of these implementations we compute

$$
\left[P_{N},\left(\begin{array}{cc}
U_{1} & 0 \\
0 & U_{2}
\end{array}\right)\right]=\left(\begin{array}{cc}
{\left[N, U_{1}\right]} & \sqrt{N N^{\prime}} U_{2}-U_{1} \sqrt{N N^{\prime}} \\
\sqrt{N N^{\prime}} U_{1}-U_{2} \sqrt{N N^{\prime}} & {\left[N^{\prime}, U_{2}\right]}
\end{array}\right),
$$

and see that that of (a) (i.e. $U_{1}=V$ and $U_{2}=1$ ) is granted if

$$
[N, V] \in \mathcal{I}_{2}, \quad(1-V) \sqrt{N N^{\prime}} \in \mathcal{I}_{2}
$$

and that of (b) $\left(U_{1}=V=U_{2}\right)$ if

$$
[N, V] \in \mathcal{I}_{2}, \quad\left[\sqrt{N N^{\prime}}, V\right] \in \mathcal{I}_{2}
$$

We can now complete the proof of parts (ii) and (iii) of the theorem. For $V=$ $\exp (\mathrm{i} \lambda Q)$, we use (a). Then Eqs. (37) hold true by Eqs. $(14,19):\left(1-\mathrm{e}^{\mathrm{i} \lambda Q}\right) \sqrt{N N^{\prime}}=$ $\left(1-\mathrm{e}^{\mathrm{i} \lambda}\right) Q \sqrt{N N^{\prime}}$. This also proves (iii). For $V=U$, we use (b), with conditions (38) holding by Eqs. $(15,24)$.

Part (iv) is readily proven as follows. Like in the pure case, the implementers are unique up to left multiplication by an element of $\pi_{N}(\mathcal{A}(\mathcal{H}))^{\prime}$ (which is larger than the multiples of the identity since the representation is reducible). Thus $\exp (\mathrm{i} \lambda \widehat{Q}) \in$ $\pi_{N}(\mathcal{A}(\mathcal{H}))^{\prime \prime}$ still implies its uniqueness up to a phase because of $(21)$.

Remark. If it were for property (ii) only, one could adopt method (b) also for $V=\exp (\mathrm{i} \lambda Q)$.

Proof of Lemma 8. The space $\mathcal{H}_{P_{N}}$ is spanned by the vectors

$$
\prod_{i=1}^{n} \pi_{P_{N}}\left(a^{\#}\left(f_{i} \oplus g_{i}\right)\right) \Omega_{P_{N}}
$$

It suffices to show that they can be approximated arbitrarily well by a sum of such vectors where, however, $g_{i}=0$. To this end, we first note that

$$
\begin{gathered}
\pi_{P_{N}}\left(a^{*}\left(P_{N}(f \oplus g)\right)\right) \Omega_{P_{N}}=0, \\
\pi_{P_{N}}\left(a\left(P_{N}^{\prime}(f \oplus g)\right)\right) \Omega_{P_{N}}=0,
\end{gathered}
$$

where $P_{N}^{\prime}=1-P_{N}$. This follows from (12) for $P_{N}$ instead of $N$, and implies in turn

$$
\begin{aligned}
\left\|\pi_{P_{N}}\left(a^{*}(f \oplus g)\right) \Omega_{P_{N}}\right\| & \leq\left\|P_{N}^{\prime}(f \oplus g)\right\|, \\
\left\|\pi_{P_{N}}(a(f \oplus g)) \Omega_{P_{N}}\right\| & \leq\left\|P_{N}(f \oplus g)\right\| .
\end{aligned}
$$


Let us first consider the case where the last factor in (39) is an annihilation operator and set $f_{n} \oplus g_{n}=: f \oplus g$. We have

$$
P_{N}(f \oplus g-\widetilde{f} \oplus 0)=\left(\begin{array}{l}
\sqrt{N} \\
\sqrt{N^{\prime}}
\end{array}\right)\left(\sqrt{N}(f-\widetilde{f})+\sqrt{N^{\prime}} g\right) .
$$

A vector $\tilde{f} \in \mathcal{H}$ is well-defined by

$$
\sqrt{N} \tilde{f}:=\sqrt{N} f+\sqrt{N^{\prime}} F(N \geq \epsilon) g,
$$

where $F(N \geq \epsilon)$ is the spectral projection for $N$ on $[\epsilon, \infty)$ and $\epsilon>0$. Thus,

$$
\left\|P_{N}(f \oplus g-\tilde{f} \oplus 0)\right\| \leq\|F(N<\epsilon) g\|
$$

can be made arbitrarily small because of $\operatorname{Ker} N=\{0\}$. If the last factor is a creation operator, the arguments proceed similarly using $\operatorname{Ker} N^{\prime}=\{0\}$. Hence the announced replacement can be performed in the last factor. After anticommuting it to the left, the claim is reduced to products with fewer factors, for which it holds by induction.

4.5. Part (v). The idea of the proof is to approximate the Bogoliubov automorphism induced by $\mathrm{e}^{\mathrm{i} \lambda Q}$ by means of inner automorphisms, as introduced in Subsect. 4.3. The generating function on the 1.h.s. of (18) then becomes computable by Lemma 4 . We present separate proofs in the pure and the mixed case. The second proof, while applying to both cases, is longer than the one we give for pure states. Both depend on Prop. 7.

Pure state. Let $F$ be a finite rank operator on $\mathcal{H}$ with $[F, N]=0$. As such, it has an implementation in the cyclic representation $\pi_{N}$; its non-uniqueness does not affect the 1.h.s. of

$$
\begin{aligned}
\left(\Omega_{N}, \widehat{U}^{*} \mathrm{e}^{\mathrm{i} \lambda \widehat{F}} \widehat{U} \mathrm{e}^{-\mathrm{i} \lambda \widehat{F}} \Omega_{N}\right) & =\left(\Omega_{N}, \widehat{U}^{*} \pi_{N}\left(\Gamma\left(\mathrm{e}^{\mathrm{i} \lambda F}\right)\right) \widehat{U} \pi_{N}\left(\Gamma\left(\mathrm{e}^{-\mathrm{i} \lambda F}\right)\right) \Omega_{N}\right) \\
& =\left(\Omega_{N}, \pi_{N}\left(\Gamma\left(U^{*} \mathrm{e}^{\mathrm{i} \lambda F} U \mathrm{e}^{-\mathrm{i} \lambda F}\right)\right) \Omega_{N}\right)
\end{aligned}
$$

on the r.h.s. we used that $\pi_{N}\left(\Gamma\left(\mathrm{e}^{\mathrm{i} \lambda F}\right)\right)$ is one possible implementation of $\mathrm{e}^{\mathrm{i} \lambda F}$ by (26) with $\mathrm{e}^{\mathrm{i} \lambda F}$ in place of $U$; the second line follows by (16), which implies $\widehat{U}^{*} \pi_{N}\left(\Gamma\left(\mathrm{e}^{\mathrm{i} \lambda F}\right)\right) \widehat{U}=$ $\pi_{N}\left(\Gamma\left(U^{*} \mathrm{e}^{\mathrm{i} \lambda F} U\right)\right)$, and by (27). Another choice for $\widehat{F}$ is fixed by

$$
\left(\Omega_{N}, \widehat{F} \Omega_{N}\right)=0 \text {, }
$$

and we may ask the same normalization for $\widehat{Q}$.

Lemma 9. There is a sequence of finite dimensional orthogonal projections $F_{n}$ such that

$$
\left[F_{n}, N\right]=0, \quad \mathrm{~s}-\lim _{n} F_{n}=Q .
$$

Proof. We note that $(N Q)^{2}=N Q$, so that $Q=N Q+N^{\prime} Q$ is an orthogonal splitting of $Q$. Let $F_{n}=F_{n}^{(1)}+F_{n}^{(2)}$, where $F_{n}^{(1)}$, resp. $F_{n}^{(2)}$, is a subprojection of $N Q$ (i.e. $F_{n}^{(1)} N Q=F_{n}^{(1)}$ ), resp. of $N^{\prime} Q$, with $F_{n}^{(1)} \stackrel{s}{\rightarrow} N Q$, and $F_{n}^{(2)} \stackrel{s}{\rightarrow} N^{\prime} Q$. Clearly, $F_{n} \stackrel{s}{\rightarrow} Q$ and

$$
\left[F_{n}^{(1)}, N\right]=\left[N^{\prime}, F_{n}^{(1)}\right]=N^{\prime} F_{n}^{(1)}-F_{n}^{(1)} N^{\prime}=N^{\prime} N Q F_{n}^{(1)}-F_{n}^{(1)} Q N N^{\prime}=0,
$$

since $N N^{\prime}=0$. The same holds for $F_{n}^{(2)}$, and thus for $F_{n}$. 
By $(42,41)$ the assumptions of Prop. 7 are satisfied for the sequence $\left(F_{n}\right)$ and its limit $Q$. Therefore,

$$
\left(\Omega_{N}, \widehat{U}^{*} \mathrm{e}^{\mathrm{i} \lambda \widehat{Q}} \widehat{U} \mathrm{e}^{-\mathrm{i} \lambda \widehat{Q}} \Omega_{N}\right)=\lim _{n \rightarrow \infty}\left(\Omega_{N}, \widehat{U}^{*} \mathrm{e}^{\mathrm{i} \lambda \widehat{F_{n}}} \widehat{U} \mathrm{e}^{-\mathrm{i} \lambda \widehat{F_{n}}} \Omega_{N}\right) .
$$

By Eqs. $(40,30,42)$ the inner product on the r.h.s. equals

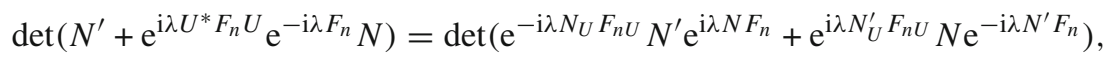

where we multiplied the determinant by

$$
1=\operatorname{det}\left(\mathrm{e}^{-\mathrm{i} \lambda N_{U} F_{n U}}\right) \cdot \operatorname{det}\left(\mathrm{e}^{\mathrm{i} \lambda N F_{n}}\right)
$$

like in the heuristic derivation (7); but unlike there, this step is now correct, since $F_{n}$ is of finite rank. We also used $\left[F_{n}, N\right]=0$. Finally, we claim that the operator under the last determinant converges to

$$
\mathrm{e}^{-\mathrm{i} \lambda N_{U} Q_{U}} N^{\prime} \mathrm{e}^{\mathrm{i} \lambda N Q}+\mathrm{e}^{\mathrm{i} \lambda N_{U}^{\prime} Q_{U}} N \mathrm{e}^{-\mathrm{i} \lambda N^{\prime} Q}=\mathrm{e}^{-\mathrm{i} \lambda N_{U} Q_{U}} N^{\prime}+\mathrm{e}^{\mathrm{i} \lambda N_{U}^{\prime} Q_{U}} N
$$

in trace class norm, i.e. the same expression with $Q$ in place of $F_{n}$. The r.h.s. is obtained using $\exp (\mathrm{i} \lambda N Q)=1+N Q(\exp (\mathrm{i} \lambda)-1)$ and $N N^{\prime}=0$. The convergence implies that of the determinants: Indeed, for $A-1, B-1 \in \mathcal{I}_{1}$, we have ([18], Lemma XIII.17.1 (d))

$$
|\operatorname{det} A-\operatorname{det} B| \leq\|A-B\|_{1} \mathrm{e}^{\left(\|A-1\|_{1}+\|B-1\|_{1}+1\right)} .
$$

Upon conjugating with $U$, it is enough to show

$$
\left\|\left(\mathrm{e}^{-\mathrm{i} \lambda N F_{n}}-\mathrm{e}^{-\mathrm{i} \lambda N Q}\right) N_{U^{*}}^{\prime}\right\|_{1} \longrightarrow 0
$$

and similarly with $N$ and $N^{\prime}$ interchanged. This operator equals $\mathrm{e}^{-\mathrm{i} \lambda}-1$ times

$$
N\left(F_{n}-Q\right) N_{U^{*}}^{\prime}=\left(F_{n}-Q\right) N N^{\prime}+\left(F_{n}-Q\right) N\left(N_{U^{*}}^{\prime}-N^{\prime}\right) .
$$

The first term vanishes, and the second tends to 0 in the trace class norm as $n \rightarrow \infty$, because of

$$
X_{n} \stackrel{s}{\longrightarrow} 0, \quad Y \in \mathcal{I}_{1} \Longrightarrow\left\|X_{n} Y\right\|_{1} \longrightarrow 0
$$

Mixed state. Let us start by proving a result analogous to Lemma 9:

Lemma 10. Let $P, Q$ be orthogonal projections in a separable Hilbert space $\mathcal{H}$ with

$$
[Q, P] \in \mathcal{I}_{1} .
$$

Then there are finite dimensional subprojections $F_{n}$ of $Q$ with

$$
\left\|\left[F_{n}-Q, P\right]\right\|_{1} \longrightarrow 0, \quad(n \rightarrow \infty) .
$$


Proof. We split $Q$ as

$$
Q=Q P Q+Q(1-P) Q \equiv L_{1}+L_{0},
$$

and observe that $\left[Q, L_{1}\right]=0$ and

$$
\begin{aligned}
& (P-1) L_{1} \in \mathcal{I}_{1}, \\
& L_{1}^{2}-L_{1}=Q P[Q, P] Q \in \mathcal{I}_{1} .
\end{aligned}
$$

By the last property, the only possible accumulation points in the spectrum of $L_{1}$ are 0 and 1. In particular, there is an $x \in(0,1)$ which is not in the spectrum. Let $Q_{1}$ be the spectral projection of $L_{1}$ associated with $(x, \infty)$. It may be represented as

$$
Q_{1}=\frac{1}{2 \pi \mathrm{i}} \oint_{\mathcal{C}}\left(z-L_{1}\right)^{-1} d z
$$

where $\mathcal{C} \subset \mathbb{C}$ is a contour encircling that part of the spectrum only. Using $\oint_{\mathcal{C}} z^{-1} d z=0$, due to $x>0$, we have

$$
\begin{aligned}
(P-1) Q_{1} & =\frac{1}{2 \pi \mathrm{i}} \oint_{\mathcal{C}}(P-1)\left(\left(z-L_{1}\right)^{-1}-z^{-1}\right) d z \\
& =\frac{1}{2 \pi \mathrm{i}} \oint_{\mathcal{C}}(P-1) L_{1}\left(z-L_{1}\right)^{-1} z^{-1} d z \in \mathcal{I}_{1}
\end{aligned}
$$

by (50). On the subspace $\operatorname{Ran} Q$, the projection $Q_{1}$, defined in terms of $L_{1}$ and $x$ is complementary to the one, $Q_{0}$, similarly defined by $L_{0}$ and $1-x$, see (49). Since $1-x>0$, we have

$$
P Q_{0} \in \mathcal{I}_{1}
$$

by analogy to (51). Let now $F_{n}^{(i)},(i=0,1)$, be a sequence of finite dimensional subprojections of $Q_{i}$ with $F_{n}^{(i)} \stackrel{s}{\rightarrow} Q_{i}$. Then

$$
\begin{aligned}
{\left[F_{n}^{(0)}-Q_{0}, P\right]=} & \left(F_{n}^{(0)}-Q_{0}\right) P-P\left(F_{n}^{(0)}-Q_{0}\right)=\left(F_{n}^{(0)}-Q_{0}\right) Q_{0} P \\
& -P Q_{0}\left(F_{n}^{(0)}-Q_{0}\right), \\
{\left[F_{n}^{(1)}-Q_{1}, P\right]=} & {\left[F_{n}^{(1)}-Q_{1}, P-1\right]=\left(F_{n}^{(1)}-Q_{1}\right) Q_{1}(P-1) } \\
& -(P-1) Q_{1}\left(F_{n}^{(1)}-Q_{1}\right),
\end{aligned}
$$

are trace class by $(51,52)$, and converge to zero in the corresponding norm by (46) and $\left\|T^{*}\right\|_{1}=\|T\|_{1}$. Thus $F_{n}=F_{n}^{(0)}+F_{n}^{(1)}$ is seen to have the stated properties.

We apply the lemma to $\mathcal{H} \oplus \mathcal{H}, P_{N}$ and $\widetilde{Q}=Q \oplus 0$ instead of $\mathcal{H}, P$ and $Q$; in this case, subprojections of $\widetilde{Q}$ are of the form $F \oplus 0$, with $F$ a subprojection of $Q$. Since

$$
\left[P_{N}, \widetilde{Q}\right]=\left(\begin{array}{cc}
{[N, Q]} & -Q \sqrt{N N^{\prime}} \\
\sqrt{N N^{\prime}} Q & 0
\end{array}\right),
$$

the hypothesis (47) of Lemma 10 is fulfilled. The claim yields

$$
\left\|\left[F_{n}-Q, N\right]\right\|_{1} \stackrel{n \rightarrow \infty}{\longrightarrow} 0
$$


as well as $\left\|\sqrt{N N^{\prime}}\left(F_{n}-Q\right)\right\|_{1} \rightarrow 0$, which however is already known by (19) and $F_{n}=F_{n} Q$. We thus have a sequence $\left(F_{n}\right)$ of unitarily implementable transformations: the conditions (37) are both fulfilled, the first one because $\left[N, \exp \left(-\mathrm{i} \lambda F_{n}\right)\right]=$ $\left[N, F_{n}\right](\exp (-\mathrm{i} \lambda)-1)$ and the second because $\left(1-\exp \left(-\mathrm{i} \lambda F_{n}\right)\right) \sqrt{N N^{\prime}}=(\exp (-\mathrm{i} \lambda)-1)$ $F_{n} Q \sqrt{N N^{\prime}}$. Moreover, the assumptions of Prop. 7 are satisfied, so that Eqs. $(43,40)$ are true again.

To complete the proof, it remains to show that

$$
\operatorname{det}\left(N^{\prime}+\mathrm{e}^{\mathrm{i} \lambda F_{n} U} \mathrm{e}^{-\mathrm{i} \lambda F_{n}} N\right) \longrightarrow \operatorname{det}\left(\mathrm{e}^{-\mathrm{i} \lambda N_{U} Q_{U}} N^{\prime} \mathrm{e}^{\mathrm{i} \lambda N Q}+\mathrm{e}^{\mathrm{i} \lambda N_{U}^{\prime} Q_{U}} N \mathrm{e}^{-\mathrm{i} \lambda N^{\prime} Q}\right) .
$$

To this end, we multiply the determinant by

$$
\operatorname{det}\left(1+F_{n U}\left(\mathrm{e}^{-\mathrm{i} \lambda N_{U}}-1\right)\right), \quad \operatorname{det}\left(1+\left(\mathrm{e}^{\mathrm{i} \lambda N}-1\right) F_{n}\right),
$$

from the left, resp. from the right. These factors would be identical to those in (44) if $F_{n}$ and $N$ commuted, which is however no longer the case. Also, their product is not 1 , but rather equals

$$
\begin{aligned}
& \operatorname{det}\left(1+F_{n U}\left(\mathrm{e}^{-\mathrm{i} \lambda N_{U}}-1\right)\right) \cdot \operatorname{det}\left(1+\left(\mathrm{e}^{\mathrm{i} \lambda N}-1\right) F_{n}\right) \\
& \quad=\operatorname{det}\left(1+\left(\mathrm{e}^{\mathrm{i} \lambda N}-1\right) F_{n}\right) \cdot \operatorname{det}\left(1+F_{n}\left(\mathrm{e}^{-\mathrm{i} \lambda N}-1\right)\right) \\
& =\operatorname{det}\left(1-F_{n}+\mathrm{e}^{\mathrm{i} \lambda N} F_{n} \mathrm{e}^{-\mathrm{i} \lambda N}\right),
\end{aligned}
$$

where

$$
\mathrm{e}^{\mathrm{i} \lambda N} F_{n} \mathrm{e}^{-\mathrm{i} \lambda N}-F_{n}=\mathrm{i} \int_{0}^{\lambda} \mathrm{e}^{\mathrm{i} s N}\left[N, F_{n}\right] \mathrm{e}^{-\mathrm{i} s N} d s \in \mathcal{I}_{1}
$$

and

$$
\left\|\mathrm{e}^{\mathrm{i} \lambda N} F_{n} \mathrm{e}^{-\mathrm{i} \lambda N}-F_{n}\right\|_{1} \stackrel{n \rightarrow \infty}{\longrightarrow} 0
$$

by $[N, Q]=0$ and (53). Therefore, (56) converges to 1 and it suffices to prove (54) with the l.h.s. multiplied by (55). The determinant becomes that of

$$
\left(1+F_{n U}\left(\mathrm{e}^{-\mathrm{i} \lambda N_{U}}-1\right)\right)\left(N^{\prime}+\mathrm{e}^{\mathrm{i} \lambda F_{n U}} \mathrm{e}^{-\mathrm{i} \lambda F_{n}} N\right)\left(1+\left(\mathrm{e}^{\mathrm{i} \lambda N}-1\right) F_{n}\right) .
$$

By means of

$$
\begin{aligned}
& \left\|\left(1+F_{n U}\left(\mathrm{e}^{-\mathrm{i} \lambda N_{U}}-1\right)\right) N^{\prime}-\mathrm{e}^{-\mathrm{i} \lambda N_{U} Q_{U}} N^{\prime}\right\|_{1} \longrightarrow 0, \\
& \left\|\mathrm{e}^{-\mathrm{i} \lambda F_{n}} N \mathrm{e}^{\mathrm{i} \lambda F_{n}}-N\right\|_{1} \longrightarrow 0,
\end{aligned}
$$

which we shall prove momentarily, we may replace (58) by

$$
\begin{aligned}
& \mathrm{e}^{-\mathrm{i} \lambda N_{U} Q_{U}} N^{\prime}\left(1+\left(\mathrm{e}^{\mathrm{i} \lambda N}-1\right) F_{n}\right)+\left(1+F_{n U}\left(\mathrm{e}^{-\mathrm{i} \lambda N_{U}}-1\right)\right) \mathrm{e}^{\mathrm{i} \lambda F_{n U}} N \mathrm{e}^{-\mathrm{i} \lambda F_{n}} \\
& \quad \times\left(1+\left(\mathrm{e}^{\mathrm{i} \lambda N}-1\right) F_{n}\right) .
\end{aligned}
$$

The claim then follows from

$$
\begin{aligned}
& \left\|N^{\prime}\left(1+\left(\mathrm{e}^{\mathrm{i} \lambda N}-1\right) F_{n}\right)-N^{\prime} \mathrm{e}^{\mathrm{i} \lambda N Q}\right\|_{1} \longrightarrow 0, \\
& \left\|N \mathrm{e}^{-\mathrm{i} \lambda F_{n}}\left(1+\left(\mathrm{e}^{\mathrm{i} \lambda N}-1\right) F_{n}\right)-N \mathrm{e}^{-\mathrm{i} \lambda N^{\prime} Q}\right\|_{1} \longrightarrow 0, \\
& \left\|\left(1+F_{n U}\left(\mathrm{e}^{-\mathrm{i} \lambda N_{U}}-1\right)\right) \mathrm{e}^{\mathrm{i} \lambda F_{n U}} N-\mathrm{e}^{\mathrm{i} \lambda N_{U}^{\prime} Q_{U}} N\right\|_{1} \longrightarrow 0 .
\end{aligned}
$$




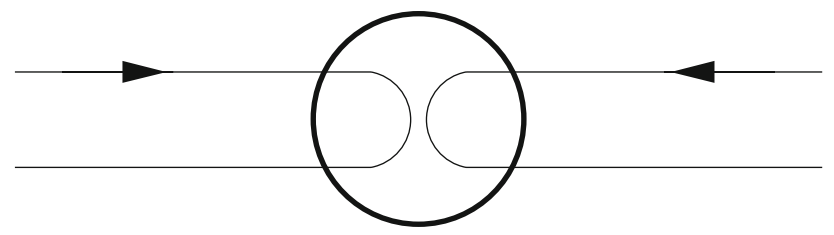

Fig. 2. A simple model with two infinite chiral leads

It remains to prove (59-63). The limit (60) follows like (57). The expression in (61) is $N^{\prime}(\exp (\mathrm{i} \lambda N)-1)\left(F_{n}-Q\right)=f(N) N N^{\prime} Q\left(F_{n}-Q\right)$, where $f(N)=N^{-1}(\exp (\mathrm{i} \lambda N)-1)$ is a bounded operator; its convergence to zero follows from (19). As for (62) we have

$$
\begin{aligned}
\mathrm{e}^{-\mathrm{i} \lambda F_{n}}\left(1+\left(\mathrm{e}^{\mathrm{i} \lambda N}-1\right) F_{n}\right) & =\left(1+\left(\mathrm{e}^{-\mathrm{i} \lambda}-1\right) F_{n}\right)\left(1+\left(\mathrm{e}^{\mathrm{i} \lambda N}-1\right) F_{n}\right) \\
& =1+\left(\mathrm{e}^{-\mathrm{i} \lambda} \mathrm{e}^{\mathrm{i} \lambda N}-1\right) F_{n}+\left(\mathrm{e}^{-\mathrm{i} \lambda}-1\right)\left[F_{n}, \mathrm{e}^{\mathrm{i} \lambda N}\right] F_{n},
\end{aligned}
$$

so that by using (57) it remains to show

$$
\left\|N\left(1+\left(\mathrm{e}^{-\mathrm{i} \lambda N^{\prime}}-1\right) F_{n}\right)-N \mathrm{e}^{-\mathrm{i} \lambda N^{\prime} Q_{\|}}\right\|_{1} \longrightarrow 0 .
$$

This, however, is just (61) with $N$ and $N^{\prime}$ interchanged. Finally, in $(59,63)$ we may, by (15), replace $N$ and $N^{\prime}$ by $N_{U}$ and $N_{U}^{\prime}$ in those places where the subscript is not already present. By passing to a unitary conjugate and adjoint, they reduce to $(61,62)$.

\section{Examples}

We illustrate the hypotheses $(14,15)$ by presenting a model in which they can be verified. The left and right portions of the single lead mentioned in Sect. 1 are replaced by two infinite leads, which are however chiral. The interaction between them occurs in a finite interval and allows particles to scatter between the leads.

Let $\mathcal{H}=L^{2}(\mathbb{R}) \oplus L^{2}(\mathbb{R})$ be the one-particle space with operators

$$
Q=\left(\begin{array}{ll}
1 & 0 \\
0 & 0
\end{array}\right), \quad N=\left(\begin{array}{cc}
\Theta(-p) & 0 \\
0 & \Theta(p)
\end{array}\right) .
$$

Here, $x \in \mathbb{R}$ is the position variable, $p=-\mathrm{i} d / d x$ the conjugate momentum, and $\Theta$ the Heaviside function. The projection $N$ describes the Fermi sea of the free Hamiltonian

$$
H_{0}=\left(\begin{array}{cc}
p & 0 \\
0 & -p
\end{array}\right)
$$

for vanishing Fermi energy. Clearly, $[Q, N]=0$.

5.1. Example 1. The example is conveniently stated by passing to another pair of conjugate variables, $E$ and $t$ : The energy $E:= \pm p$ yields the spectral representation of $H_{0}$ in multiplication form,

$$
H_{0}=\left(\begin{array}{cc}
E & 0 \\
0 & E
\end{array}\right)
$$


while the operator

$$
T=\left(\begin{array}{cc}
-x & 0 \\
0 & x
\end{array}\right)=:\left(\begin{array}{cc}
t & 0 \\
0 & t
\end{array}\right),
$$

represents, due to $\mathrm{i}\left[H_{0}, T\right]=-1$, the time $t$ of passage at $x=0$ of a freely moving particle, which is presently elsewhere. In this example only, the meaning of $t$ is therefore that of a dynamical variable, and not that of the parameter governing evolution.

Rather than specifying an interacting Hamiltonian, we model the scattering process by directly giving the propagator $U$ for the time interval under consideration. We assume it to be given by a unitary multiplication operator $U(t)$ with $U(t)-1$ of compact support, see Remark 1 in Sect. 3.

Such a simple kind of evolution should be seen as an effective description in the adiabatic limit and in the interaction picture. The passage across the interaction region maps the incoming state to the outgoing one by means of a scattering matrix which, in the limit of low frequencies $\omega$, is that of the static scatterer in effect at time $t, S(t)$. In the same limit, only electrons within an interval $\sim \hbar \omega$ of the Fermi energy ought to matter for transport. Thus, $U(t)=S(t, 0)$, where the $2 \times 2$-matrix $S(t, E)$ is the fiber of $S(t)$ at energy $E$. For a more thorough justification, see [6,3].

Proposition 11. Suppose $U-1 \in M_{2}\left(C_{0}^{\infty}\left(\mathbb{R}_{t}\right)\right)$. Then $[N, U] \in \mathcal{I}_{1}$.

Here $M_{2}(X)$ are the $2 \times 2$ matrices with entries in $X$.

Proof. We may rename $U(t)-1$ by $U(t)$ without loss. By the assumption we may write $U=f U$, where $f=f(t)$ satisfies $f \in C_{0}^{\infty}\left(\mathbb{R}_{t}\right)$, too. Then $[N, U]=f[N, U]+$ $[N, f] U$, with

$$
f[N, U]=f(E+\mathrm{i})^{-1} \cdot(E+\mathrm{i})[N, U],
$$

and similarly for the second term. We claim that both factors are Hilbert-Schmidt and hence their product trace class. The first one is, because the functions $f$ and $g(E) \equiv(E+$ i) $)^{-1}$ are in $L^{2}\left(\mathbb{R}_{t}\right)$, resp. $L^{2}\left(\mathbb{R}_{E}\right)$. As for the second one, we note that $N=\Theta(-E) \otimes 1_{2}$, whence $[N, U]$ has matrix entries $\left[\Theta(-E), U_{i j}\right]$. That leads to integral operators $K$ acting merely on $L^{2}\left(\mathbb{R}_{E}\right)$ with kernels

$$
K\left(E, E^{\prime}\right)=(E+\mathrm{i}) \widehat{U}_{i j}\left(E^{\prime}-E\right)\left(\Theta(-E)-\Theta\left(-E^{\prime}\right)\right) .
$$

They are supported where $\operatorname{sgn} E=-\operatorname{sgn} E^{\prime}$ and satisfy

$$
\begin{aligned}
\iint\left|K\left(E, E^{\prime}\right)\right|^{2} d E d E^{\prime}= & \int_{0}^{\infty} \int_{0}^{\infty}\left|E^{\prime \prime}+\mathrm{i}\right|^{2}\left(\left|\widehat{U}_{i j}\left(E^{\prime}+E^{\prime \prime}\right)\right|^{2}+\left|\widehat{U}_{i j}\left(-E^{\prime}-E^{\prime \prime}\right)\right|^{2}\right) \\
& \times d E d E^{\prime \prime},
\end{aligned}
$$

which is finite. Thus, the corresponding operator is in $\mathcal{I}_{2}$.

By contrast, but under the same assumption as in the proposition, the operator $\left(Q_{U}-\right.$ $Q) N$ may fail to be trace class. By (4), this shows the need for regularizing (3). Indeed, we may arrange for a $\psi \in \mathcal{H}$ and $U$ such that $\left(Q_{U}-Q\right) \psi \neq 0$. The sequence $\psi_{n}=$ $\exp (\mathrm{i} n T) \psi$ tends to zero weakly. Using $\Theta(-E) \exp (\mathrm{i} n t)=\exp (\mathrm{i} n t) \Theta(n-E)$ and $\Theta(n-E) \stackrel{s}{\rightarrow} 1$, we have $\left\|N \psi_{n}-\psi_{n}\right\| \rightarrow 0$ and, since $Q, U$ are multiplication operators in $t,\left\|\left(Q_{U}-Q\right) N \psi_{n}\right\| \rightarrow\left\|\left(Q_{U}-Q\right) \psi\right\| \neq 0$. As a result, $\left(Q_{U}-Q\right) N$ 
is not even compact. The argument just given may be summarized in physical terms as follows: Whatever contribution to transport, as signified by $\left(Q_{U}-Q\right) N$, comes from one energy in the Fermi sea, it is repeated at all such energies, because the evolution $U$ proceeds with the same velocity \pm 1 at all energies.

It should be remarked that $[N, U]$ may fail to be in $\mathcal{I}_{2}$ if, unlike in Prop. 11, $U(t)$ attains different limits at $t \rightarrow \pm \infty$. This fact has been pointed out in [12] in slightly different terms as a manifestation of the orthogonality catastrophe. Consider for instance a potential drop $V(t)$ of finite duration being applied between the leads, with $\int_{-\infty}^{\infty} V(t) d t \notin 2 \pi \mathbb{Z}$. That situation can be modeled in the context of the present example by means of a vector potential, where it gives rise to the catastrophe. The same physical situation is however tame in the context of the next example.

5.2. Example 2. Here we specify a time-dependent perturbation of (65), $H(t)=H_{0}+$ $V(t)$, where $V(t)$ is multiplication by a $2 \times 2$ matrix $V(t, x)$. Let $U=U\left(t_{2}, t_{1}\right)$ be the propagator for $H(t)$ between times $t_{1}$ and $t_{2}$.

Proposition 12. Suppose $V(t, \cdot), \partial_{t} V(t, \cdot) \in M_{2}\left(C_{0}^{\infty}\left(\mathbb{R}_{x}\right)\right)$. Then $[N, U] \in \mathcal{I}_{2}$.

Note that the commutator is claimed to be Hilbert-Schmidt only, which covers only the statements (ii-iv) of Theorem 1.

Proof. By [23], Lemma 4 or [20], Thm. 2.8 it suffices to show that the statement holds true for the first term in the Dyson expansion of $U$, i.e. for

$$
\tilde{U}\left(s_{2}, s_{1}\right)=-\mathrm{i} \int_{s_{1}}^{s_{2}} \mathrm{e}^{\mathrm{i} H_{0} t} V(t) \mathrm{e}^{-\mathrm{i} H_{0} t} d t
$$

with estimates uniform in the sub-interval $\left[s_{1}, s_{2}\right] \subset\left[t_{1}, t_{2}\right]$. By writing

$$
V(t)=\left(\begin{array}{ll}
V_{++}(t) & V_{+-}(t) \\
V_{-+}(t) & V_{--}(t)
\end{array}\right)
$$

the kernel of $[N, V(t)]$ in momentum space becomes

$$
\begin{aligned}
& {[N, V(t)]\left(p, p^{\prime}\right)} \\
& \quad=\left(\begin{array}{cc}
\hat{V}_{++}\left(t, p-p^{\prime}\right)\left(\Theta(-p)-\Theta\left(-p^{\prime}\right)\right) & \hat{V}_{+-}\left(t, p-p^{\prime}\right)\left(\Theta(-p)-\Theta\left(p^{\prime}\right)\right) \\
\hat{V}_{-+}\left(t, p-p^{\prime}\right)\left(\Theta(p)-\Theta\left(-p^{\prime}\right)\right) & \hat{V}_{--}\left(t, p-p^{\prime}\right)\left(\Theta(p)-\Theta\left(p^{\prime}\right)\right)
\end{array}\right) .
\end{aligned}
$$

The diagonal contributions are in $\mathcal{I}_{2}$ without recourse to the integration (66). For instance,

$$
\iint d p d p^{\prime}\left|\hat{V}_{--}\left(t, p-p^{\prime}\right)\right|^{2}\left|\Theta(p)-\Theta\left(p^{\prime}\right)\right|=\int_{-\infty}^{\infty} d u|u|\left|\hat{V}_{--}(t, u)\right|^{2}<\infty .
$$

The off-diagonal contributions improve once the time integral is performed. We compute it by parts and obtain, for instance, the kernel

$$
\begin{aligned}
& -\mathrm{i} \int_{s_{1}}^{s_{2}} \hat{V}_{+-}\left(t, p-p^{\prime}\right) \mathrm{e}^{\mathrm{i}\left(p+p^{\prime}\right) t} d t \\
& =-\left.\frac{\mathrm{e}^{\mathrm{i}\left(p+p^{\prime}\right) t}-1}{p+p^{\prime}} \hat{V}_{+-}\left(t, p-p^{\prime}\right)\right|_{s_{1}} ^{s_{2}}+\int_{s_{1}}^{s_{2}} \frac{\mathrm{e}^{\mathrm{i}\left(p+p^{\prime}\right) t}-1}{p+p^{\prime}} \partial_{t} \hat{V}_{+-}\left(t, p-p^{\prime}\right) d t,
\end{aligned}
$$


times $\Theta(-p)-\Theta\left(p^{\prime}\right)$. The boundary terms are separately in $\mathcal{I}_{2}$, since their corresponding square norm is

$$
\begin{aligned}
& 4 \iint d p d p^{\prime} \frac{\sin ^{2}\left(\left(p+p^{\prime}\right) s_{i} / 2\right)}{\left(p+p^{\prime}\right)^{2}}\left|\hat{V}_{+-}\left(s_{i}, p-p^{\prime}\right)\right|^{2}\left|\Theta(-p)-\Theta\left(p^{\prime}\right)\right| \\
& \quad=4 \int_{-\infty}^{\infty} d u \frac{\sin ^{2}\left(u s_{i} / 2\right)}{u^{2}} \int_{-|u| / 2}^{|u| / 2} d v\left|\hat{V}_{+-}\left(s_{i}, v\right)\right|^{2} \leq \pi\left|s_{i}\right|\left\|V_{+-}\left(s_{i}\right)\right\|_{2}^{2}
\end{aligned}
$$

By the same estimate, but with $\partial_{t} V_{+-}(t)$ in place of $V_{+-}\left(s_{i}\right)$, also the integrand in (67) is in $\mathcal{I}_{2}$.

We recall that in $[23,20]$ the implementation of the propagator of a time-dependent Dirac Hamiltonian was studied, of which the above $H(t)$ is the 1-dimensional version. In larger dimensions, as considered there, the implementability is ensured only in some cases.

We remark that by the method used in Example 1 one can show that diagonal perturbations lead to $[N, U] \in \mathcal{I}_{1}$, but not for off-diagonal ones.

Acknowledgements. We would like to thank H. Araki, P. Deift, G. Dell'Antonio, G. Kottanattu, G. Lesovik and W. de Roeck for discussions. We also thank the Erwin Schrödinger Institute (Vienna) and the Lewiner Institute for Theoretical Physics at the Technion (Haifa) for hospitality.

\section{References}

1. Araki, H.: Bogoliubov automorphisms and Fock representations of the canonical anticommutation relations. Contemp. Math. 62, 23-141 (1987)

2. Araki, H., Wyss, W.: Representations of canonical anticommutation relations. Helv. Phys. Acta 37, 136159 (1964)

3. Avron, J.E., Elgart, A., Graf, G.M., Sadun, L., Schnee, K.: Adiabatic charge pumping in open quantum systems. Comm. Pure Appl. Math. 57, 528-561 (2004)

4. Baumgärtel, H., Jurke, M., Lledó, F.: Twisted duality of the CAR-algebra. J. Math. Phys. 43, 4158-4179 (2002)

5. Bratteli, O., Robinson, D.W.: Operator Algebras and Quantum Statistical Mechanics I. Berlin-HeidelbergNew York: Springer-Verlag, 1979

6. Büttiker, M., Thomas, H., Prêtre, A.: Current partition in multi-probe conductors in the presence of slowly oscillating external potentials. Z. Phys. B 94, 133-137 (1994)

7. Doplicher, S., Haag, R., Roberts, J.E.: Fields, observables and gauge transformations I. Commun. Math. Phys. 13, 1-23 (1969)

8. Ivanov, D.A., Lee, H.W., Levitov, L.S.: Coherent states of alternating current. Phys. Rev. B 56, 68396850 (1997)

9. Jakšić, V., Pillet, C.-A.: Mathematical theory of non-equilibrium quantum statistical mechanics. J. Stat. Phys. 108, 787-829 (2002)

10. Klich, I.: Full counting statistics: An elementary derivation of Levitov's formula. In: Yu. V. Nazarov, Ya. M. Blanter, eds, Quantum Noise. Dordrecht: Kluwer, 2003

11. Levitov, L.S.: Counting statistics of charge pumping in open systems. http://arxiv.org/list/cond-mat/ 0103617,2001

12. Levitov, L.S., Lee, H.W., Lesovik, G.B.: Electron counting statistics and coherent states of electric current. J. Math. Phys. 37, 4845-4866 (1996)

13. Levitov, L.S., Lesovik, G.B.: Charge distribution in quantum shot noise. JETP Lett. 58, 230-235 (1993)

14. Lundberg, L.-E.: Quasi-free 'second quantization'. Commun. Math. Phys. 50, 103-112 (1976)

15. Muzykanskii, B.A., Adamov, Y.: Scattering approach to counting statistics in quantum pumps. Phys. Rev. B 68, 155304-155313 (2003)

16. Pilgram, S., Büttiker, M.: Statistics of charge fluctuations in chaotic cavities. Phys. Rev. B 67, 235308 (2003) 
17. Powers, R.T., Størmer, E.: Free states of the canonical anticommutation relations. Commun. Math. Phys. 16, 1-33 (1970)

18. Reed, M., Simon, B.: Methods of Modern Mathematical Physics: IV, Analysis of Operators. New York: Academic Press, 1978

19. De Roeck, W.: Large deviation generating function for energy transport in the Pauli-Fierz model. http:// arxiv.org/abs/0704.3400v3, 2007

20. Ruijsenaars, S.N.M.: Charged particles in external fields. I. Classical theory. J. Math. Phys. 18, 720-737 (1977)

21. Schönhammer, K.: Full counting statistics for noninteracting fermions: Exact results and the LevitovLesovik formula. Phys. Rev. B 75, 205329 (2007)

22. Seiler, E.: Schwinger functions for the Yukawa model in two dimensions with space-time cutoff. Commun. Math. Phys. 42, 163-182 (1975)

23. Seiler, R.: Quantum theory of particles with spin zero and one half in external fields. Commun. Math. Phys. 25, 127-151 (1972)

24. Shale, D., Stinespring, W.F.: Spinor representations of infinite orthogonal groups. J. Math. Mech. 14, 315-322 (1965)

Communicated by M. Aizenman 